\title{
Proposição de um conjunto de metadados para descrição de arquivos fotográficos considerando a Nobrade e a Sepiades
}

\author{
Proposition of a metadata group for photographic archives \\ description considering the Nobrade and the Sepiades norms
}

Neiva PAVEZI'

Daniel FLORES²

Carlos Blaya PEREZ ${ }^{3}$

\section{RESUMO}

A diversidade de ferramentas elaboradas e/ou utilizadas individualmente pelas várias instituições que custodiam acervos fotográficos e, por outro lado, as iniciativas de padronização da descrição desses acervos refletem um aspecto do panorama atual das discussões na área arquivística. Diante disso, e considerando a função maior dos arquivos, que é a difusão do acervo, este artigo tem por objetivo apresentar uma proposta de um conjunto de metadados para a descrição de arquivos fotográficos considerando as normas Nobrade e Sepiades. Acreditamos que essa reflexão contribuirá para as discussões que envolvam a normatização da descrição arquivística de fotografias, visando ao intercâmbio de informações de acervos fotográficos sem barreiras institucionais, territoriais, linguísticas ou operacionais.

Palavras-chave: descrição; arquivo fotográfico; Nobrade; Sepiades.

\begin{abstract}
The diversity of tools prepared and/or used individually by the several institutions that keep photographic collections on the one hand and the initiatives of a standard description pattern of those photographic collections on the other hand reflect an aspect of the current panorama of the discussions in the archives area. In view of that and considering the major function of the archives, which is the diffusion of the collection, this article aims to present a proposal of a metadata group for photographic archives description, considering the Nobrade and the Sepiades norms. It is believed that reflection will contribute to the discussions that involve the rules of the photographic archives description seeking the exchange of information of photographic collections without institutional, territorial, linguistic or operational barriers.

Keywords: description; photographic archives; Nobrade; Sepiades.

1 Arquivista do Departamento de Arquivo Geral/Universidade Federal de Santa Maria - UFSM. Tutora EAD do curso de Especialização em Gestão em Arquivos/UFSM. Mestranda em Patrimônio Cultural/Universidade Federal de Santa Maria - UFSM. Correspondência para/Corresponde to: N. PAVEZI. E-mail: <neivapavezi@yahoo.com.br>.

2 Professor do Curso de Arquivologia/Universidade Federal de Santa Maria - UFSM. Doutor em Documentação pela Universidade de Salamanca/ES.

3 Professor do Curso de Arquivologia/Universidade Federal de Santa Maria - UFSM. Doutor em Documentação pela Universidade de Salamanca/ES.

Recebido em 17/3/2009 e aceito para publicação em: 30/9/2009.
\end{abstract}




\section{INTRODUÇÃO}

acesso aos arquivos é estimulado e facilitado ao usuário através de um instrumento de pesquisa que pode oferecer informações resumidas ou detalhadas sobre o conteúdo documental do seu acervo. A produção de um instrumento de pesquisa de arquivos requer o planejamento dos elementos descritivos. Esse planejamento envolve tanto a ferramenta de descrição, seja ela manual ou informatizada, quanto a observância de um conjunto de normas e regras que a discipline e uniformize. Uma descrição bem planejada também oferece uma oportunidade para registrar informações administrativas sobre o acervo, sua condição física, e quaisquer restrições de acesso similares de gestão da informação.

Através da produção de um instrumento de pesquisa consoante com o objetivo principal de promover a difusão pretende-se ampliar a abrangência da utilização do acervo fotográfico da Universidade Federal de Santa Maria (UFSM). Para este foi realizado um estudo com o objetivo de pensar os elementos de descrição para arquivos fotográficos a partir das normas e modelos vigentes. Este estudo foi desenvolvido no âmbito do programa de pós-graduação profissionalizante em Patrimônio Cultural da UFSM.

$\mathrm{Na}$ condução da pesquisa empregou-se a pesquisa em fontes primárias (documentação arquivística da instituição), a observação direta e a exploração bibliográfica dos referenciais teóricos e normativos referentes à descrição arquivística e de fotografias.

A documentação fotográfica da UFSM é formada por negativos, diapositivos e cópias em papel, originada pela instituição no exercício da função de divulgação de suas atividades administrativas e de ensino, pesquisa e extensão. $\bigcirc$ instrumento de descrição de fotografias foi proposto a partir do estudo e análise da Norma Brasileira de Descrição - Nobrade (Brasil, 2006) e do Modelo para Descrição de Coleções Fotográficas (Sepiades) proposto pela Comunidade Européia.

Este artigo apresenta resultados parciais desse projeto. Inicia com uma breve apresentação da instituição e do acervo e, em seguida, expõe as principais características da Nobrade e da Sepiades. Na seqüência relata-se o estudo comparativo realizado entre as duas ferramentas de referência e apresenta-se os resultados parciais obtidos. Essa é a proposta inicial de um conjunto de metadados para a descrição de arquivos fotográficos elaborado para ser utilizado e testado no arquivo fotográfico da UFSM.

\section{O ACERVO FOTOGRÁFICO DA UNIVERSIDADE FEDERAL DE SANTA MARIA}

A primeira universidade construída numa pequena cidade do interior do Brasil foi criada no estado do Rio Grande do Sul em 14 de dezembro de 1960, pela Lei $n^{\circ}$ 3834-C do Governo Federal e se chamava apenas Universidade de Santa Maria (USM). Inicialmente a USM reuniu as faculdades, já existentes, de Farmácia, Medicina, Odontologia, Politécnica, Ciências Políticas e Econômicas, Agronomia, Veterinária, Direito, Belas Artes, duas de Filosofia, Ciências e Letras e a Escola Superior de Enfermagem.

Quase cinco anos depois, a Lei $n^{\circ} 4.759$, de 20 de agosto de 1965, denominou e qualificou as universidades federais e a Universidade de Santa Maria (USM) passou a se chamar Universidade Federal de Santa Maria (UFSM).

A atual estrutura, determinada pelo Estatuto da Universidade, aprovado pela Portaria Ministerial n. 801, de 27 de abril de 2001, e publicado no Diário Oficial da União em 30 de abril do mesmo ano, estabelece a constituição de oito Unidades Universitárias: Centro de Ciências Naturais e Exatas, Centro de Ciências Rurais, Centro de Ciências da Saúde, Centro de Educação, Centro de Ciências Sociais e Humanas, Centro de Tecnologia, Centro de Artes e Letras e Centro de Educação Física e Desportos. São considerados órgãos suplementares centrais a Coordenadoria de Comunicação Social e o Departamento de Arquivo Geral, dentre outros.

A Coordenadoria de Comunicação Social é a unidade administrativa responsável pela produção da documentação objeto deste estudo. Tem o propósito de estabelecer e implementar uma política global de comunicação para a UFSM, executar ações estratégicas e formular diagnósticos sobre a imagem da Instituição na comunidade. Enfim, deve contribuir significativamente para dar uma maior divulgação às ações educacionais e científicas da UFSM na imprensa. Foi instituída pela Resolução do Conselho Universitário $n^{\circ}$ 016/98, de 30/12/1998, de acordo com o parecer $n^{\circ}$ 013/98, da Comissão de Legislação e Regimentos, absorvendo a infra-estrutura da Rádio Universidade e 
da Assessoria de Imprensa do Gabinete do Reitor. É um órgão suplementar central diretamente subordinada ao Gabinete do Reitor (Universidade..., 1988).

Na execução de suas funções administrativas, essa Coordenadoria produz e utiliza registros fotográficos. Esses documentos (fotografias) são largamente utilizados nos seus vários produtos finais como a edição do jornal da UFSM, fomento da Seção de Notícias da UFSM no portal UFSM na internet, produção de notícias para outros veículos de comunicação fora da Universidade e atendimento aos veículos que procuram a instituição.

Depois da utilização dessa documentação fotográfica para os fins administrativos a que se destina, ela é recolhida ao Arquivo Permanente do Departamento de Arquivo Geral da universidade. Desta forma o atual acervo fotográfico é constituído pelas fotografias produzidas na instituição, pela Coordenadoria de Comunicação social.

\section{O Departamento de Arquivo Geral}

Departamento de Arquivo Geral (DAG) é o órgão responsável pela custódia e tratamento arquivístico da documentação oriunda das atividades dos órgãos administrativos e das unidades de ensino, pesquisa e extensão que compõem a universidade. Tratase de um órgão executivo da Administração superior, subordinada diretamente ao Gabinete do Reitor e vinculada à Pró-Reitoria de Administração, de acordo com a Resolução nº 0007/90, (Universidade..., 1990), na gestão do Reitor Tabajara Gaúcho da Costa.

Na Proposta de Regimento Interno Arquivo Geral, Capítulo V das Disposições Gerais consta:

Art. $23^{\circ}$ - É patrimônio documental da UFSM toda a documentação oficial e técnica produzida ou recebida na Instituição, no desempenho de suas atividades administrativas, de ensino, pesquisa e extensão

Art. $24^{\circ}$ - Os documentos que compõem os arquivos especiais e especializados, tais como fotografias, slides, negativos, recortes de jornais, filmes, fitas magnéticas, plantas de engenharia, arquivos médicos, etc. devem receber tratamento especializado tanto para seu arquivamento como para sua conservação. (Universidade..., 2001).
O DAG possui, entre outros setores, o Arquivo Permanente, que tem a função de reunir, organizar, tratar e dar condições de acesso ao acervo arquivístico institucional. No Setor de Arquivo Permanente, o conjunto documental fotográfico está armazenado separadamente e recebe tratamento arquivístico diferenciado. Esse processo de conscientização teve início em 1987 quando foram realizadas as primeiras intervenções arquivísticas por meio da realização de estágio dos estudantes de Arquivologia. Desde então, vários projetos foram desenvolvidos no setor, visando à melhoria do acondicionamento, descrição e disseminação dessa documentação fotográfica.

\section{A legislação que rege as atividades do DAG}

Sendo a UFSM uma instituição federal autárquica, as atividades arquivísticas realizadas no DAG seguem as orientações do Sistema de Gestão de Documentos de Arquivo (SIGA) criado por força do Decreto $n^{\circ} 4.915$, de 12 de dezembro de 2003, e também as resoluções do Conselho Nacional de Arquivos (CONARQ).

O SIGA tem por objetivos:

- Garantir ao cidadão e aos órgãos e entidades da Administração Pública Federal, de forma ágil e segura, o acesso aos documentos de arquivo e às informações neles contidas, resguardados os aspectos de sigilo e as restrições administrativas ou legais;

- Integrar e coordenar as atividades de gestão de documentos de arquivo desenvolvidas pelos órgãos setoriais e seccionais que o integram;

- Disseminar normas relativas à gestão de documentos de arquivo;

- Racionalizar a produção da documentação arquivística pública;

- Racionalizar e reduzir os custos operacionais e de armazenagem da documentação arquivística pública;

- Preservar o patrimônio documental arquivístico da Administração Pública Federal;

- Articular-se com os demais sistemas que atuam direta ou indiretamente na gestão da informação pública federal. 
O Arquivo Nacional, criado em 1838, é o órgão central do SIGA, da Administração Pública Federal. Integrante da estrutura básica da Casa Civil da Presidência da República, está diretamente subordinado à Secretaria Executiva. Tem por finalidade implementar e acompanhar a política nacional de arquivos, definida pelo Conselho Nacional de Arquivos, por meio da gestão, do recolhimento, do tratamento técnico, da preservação e da divulgação do patrimônio documental do país, garantindo pleno acesso à informação, visando a apoiar tanto as decisões governamentais de caráter político-administrativo quanto o cidadão na defesa de seus direitos, assim como incentivar a produção de conhecimento científico e cultural.

O CONARQ é um órgão colegiado, vinculado ao Arquivo Nacional da Casa Civil da Presidência da República, que tem por finalidade não apenas definir a política nacional de arquivos públicos e privados, como órgão central de um Sistema Nacional de Arquivos, mas também exercer orientação normativa visando à gestão documental e à proteção especial aos documentos de arquivo.

A Portaria n ${ }^{\circ} 56$, de 30/09/2001, do CONARQ, criou a Câmara Técnica de Normalização da Descrição Arquivística (CTNDA) com a finalidade de propor uma norma brasileira em conformidade com a Norma Geral Internacional para a Descrição Arquivística (ISAD(G)), e a Norma Internacional de Registro de Autoridade Arquivística para Entidades Coletivas, Pessoas e Famílias (ISAAR (CPF)). O resultado foi a criação e divulgação da Norma Brasileira de Descrição Arquivística (Nobrade), em 2006. A Nobrade é resultado da insistência do Comitê de Normas de Descrição $(C N D)^{4}$ para a necessidade de cada país refletir sobre sua realidade e criar normas próprias, devido ao alto grau de generalidade da ISAD(G), que define apenas a macroestrutura da descrição e deixa a definição quanto aos procedimentos específicos para outras esferas de decisão, sejam nacionais ou institucionais.

\section{INSTRUMENTOS DE REFERÊNCIA PARA A DESCRIÇÃO ARQUIVÍSTICA DE FOTOGRAFIAS: NOBRADE E SEPIADES}

Atualmente, existem diversas iniciativas visando à cooperação internacional entre as várias instituições que custodiam acervos. Também está se ampliando a utilização da documentação fotográfica como subsídio para a pesquisa em diversas áreas e, ao mesmo tempo, não há um modelo padronizado de descrição para materiais fotográficos. Normalmente, as instituições criam os seus próprios modelos, adaptam os já existentes ou utilizam modelos que não são especificamente concebidos para descrever coleções de fotografias. Nesses casos é comum a alteração de regras de determinados elementos de modelos descritivos para incluir uma descrição adequada da coleção fotográfica. Além disso, é preciso acompanhar também a evolução tecnológica que inclui, entre os suportes fotográficos tradicionais, também os documentos fotográficos digitais e eletrônicos. Diante disso fica evidente a importância de uma descrição confiável e um conjunto padronizado de elementos para o intercâmbio de informações entre instituições na Internet. Ainda, pretende-se aqui trazer uma proposta inicial onde será apresentado um conjunto de metadados para a descrição de arquivos fotográficos que possibilite também a gestão do arquivo fotográfico.

Igualmente importantes e urgentes são as iniciativas em relação à preservação dessas informações. Porém esse tema não será abordado aqui.

As principais características da Nobrade e da Sepiades e os referenciais teóricos utilizados para a construção dessa proposta serão explicitados a seguir.

\section{Norma brasileira de descrição arquivística - Nobrade}

A Norma Brasileira de Descrição Arquivística (Nobrade) estabelece diretivas para a descrição no Brasil de documentos arquivísticos, compatíveis com as normas internacionais em vigor, ISAD (General..., 2000) e ISAAR (CPF), e tem em vista facilitar o acesso e o intercâmbio de informações em âmbito nacional e internacional. A Nobrade tem por objetivo estruturar a informação a partir de elementos de descrição comuns, buscando interferir o mínimo possível na forma final em que as descrições são apresentadas. A decisão acerca dos recursos utilizados para a descrição, bem como o formato final de seus instrumentos de pesquisa é de livre escolha de cada entidade custodiadora e de seus profissionais, desde que contenha os elementos de descrição obrigatórios.

A Nobrade orienta genericamente a descrição dos acervos arquivísticos brasileiros e tem particular aplicação nos acervos formados por documentação

${ }^{4}$ Comissão criada no âmbito do Conselho Internacional de Arquivos (CIA), em 1989. 
textual. Especificamente no que se refere à fotografia, está aberta para o acréscimo dos elementos descritivos que sejam fundamentais, visando à posterior recuperação dessa documentação.

É nesse contexto que os elementos de descrição propostos no modelo Sepiades foram analisados e inseridos nessa proposição de um novo conjunto de metadados para a descrição de arquivos fotográficos.

Dentre as características da norma, destacam-se:

- Descrição do geral para o particular, cujo objetivo é representar o contexto e a estrutura hierárquica do fundo e suas partes componentes;

- Informação relevante para o nível de descrição, visando a representar com rigor o contexto e o conteúdo da unidade de descrição;

- Relação entre descrições, para explicitar a posição da unidade de descrição na hierarquia;

- Não repetição da informação, com o objetivo de evitar redundância de informação em descrições hierarquicamente relacionadas;

- Consideração de seis principais níveis de descrição (acervo da entidade custodiadora, fundo ou coleção, seção, série, dossiê ou processo, item documental), podendo ser admitidos níveis intermediários (acervo da subunidade custodiadora, subseção e subsérie);

- Não obrigatoriedade da implementação de todos os níveis, porém o nível de descrição 5 tem como requisito a existência do nível 4;

- Previsão da existência de oito áreas de descrição que totalizam 28 elementos. Em relação à $\operatorname{ISAD}(G)$, foi incluída a área oito (pontos de acesso e descrição de assuntos) e dois elementos de descrição (6.1 e 8.1);

- Apresentação de todas as regras e procedimentos a serem adotados na descrição de cada elemento.

\section{SEPIA Data Element Set - Sepiades}

Modelo Sepiades é resultado de um projeto do programa Europeu de Salvaguarda de Imagens
Fotográficas para Acesso (Safeguarding European Photographic Images for Access - SEPIA) cujo objetivo é definir o papel das novas tecnologias em um contexto de preservação em longo prazo das coleções fotográficas históricas. O programa SEPIA (Safeguarding..., 2000) reuniu representantes de diferentes tipos de instituições como bibliotecas, arquivos e museus, e institutos de investigação. É um modelo que pode ser usado para descrever coleções fotográficas e que pode ser utilizado para descrever as fotografias mais detalhadamente. Basicamente, deve funcionar ao lado de modelos descritivos já existentes, mas também pode ser usada como uma ferramenta distinta e independente.

modelo não pretende ser uma definição rígida de elementos, e apenas alguns elementos são considerados altamente recomendáveis. As principais características da Sepiades são: a descrição multinível - baseada na ISAD(G) e no Fotios (modelo descritivo holandês para materiais fotográficos, desenvolvido pela Sociedade Holandesa de Fotografia) -, a hierarquia personalizável, o conjunto padronizado de elementos, a descrição detalhada das partes fotográficas e a possibilidade de funcionar junto ou independente de outra ferramenta.

Simultaneamente a este relatório consultivo, em estreita cooperação com os grupos de trabalho, uma ferramenta de software foi desenvolvida pelo Netherlands Institute for Scientific Information Services (NIWI). Esta ferramenta implementa o modelo como foi formulado no relatório. Suas principais características são: descrição multinível, multiplataforma desenvolvida em Java, armazenamento de registros em formato XML, função de exportação Dublin Core, função de busca e recuperação, implementação do Protocolo de Open Archives Initiative Metadata Harvesting (OAI-PMH), e Open Source.

Para fins desta pesquisa foi estudado e analisado o modelo descritivo apresentado no relatório consultivo pelo grupo de trabalho do programa SEPIA. O software desenvolvido posteriormente não é objeto deste artigo

Dentre as recomendações para a catalogação de coleções fotográficas do modelo Sepiades apresentado no relatório consultivo do grupo de trabalho SEPIA, consta que:

- A estrutura da hierarquia é determinada pelo catalogador/descritor. $\bigcirc$ nível principal é o institute, que identifica a instituição 
custodiadora do acervo; o nível collection compreende um grupo de objetos produzidos por um indivíduo ou organização (identificação do produtor); o nível grouping considera um conjunto de imagens físicas e que pode ser uma subdivisão de uma collection ou de um outro grouping; e o nível single item é o componente de uma collection ou de um grouping;

- Nível single item diferencia imagem física de visual, ou seja, cada cena visível é registrada uma única vez e pode ser conectada a diferentes suportes que possam existir;

- Possui o nível acquisition, cujo objetivo é registrar como cada imagem ingressou na instituição, quem foi o proprietário anterior e o que aconteceu nesse período (proveniência);

- Cada nível possui um conjunto de descritores subdivididos em administration, provenance e material, onde administration possui elementos relevantes para a gestão administrativa, provenance agrupa informações a respeito da origem da unidade descrita e material reúne informações referentes às características das imagens visuais e físicas;

- Considera como elementos descritivos especiais name, date e geographical location. O elemento name inclui os campos function (para descrever a relação de alguém ou alguma coisa com a fotografia) e type (indica a que ou a quem a informação se refere). O elemento geographical location inclui os campos specification, role e additional information (recomendado para conectar nomes que foram alterados, ou fazer referência a um tesauro utilizado). O elemento date pode se referir a várias coisas como data da captura da imagem, do processamento do suporte e da publicação da imagem. Inclui os campos specification, role e status para informar a condição;

- Para normatizar o preenchimento dos campos, propõe a utilização das normas ISO
3166 (para código do país) e ISO 8601 (para data e hora. O relatório final do grupo de trabalho SEPIA em modelo descritivo para coleções fotográficas detalha os procedimentos a serem adotados na descrição de cada campo.

\section{O estudo comparativo dos elementos descritivos Nobrade e Sepiades}

A metodologia adotada para propor um conjunto de metadados pela interação dos elementos descritivos do modelo Sepiades e Nobrade iniciou pela leitura das respectivas referências e análise dos objetivos, regras e recomendações de cada elemento descritivo. Com esse conhecimento foi possível, inicialmente, estabelecer correspondências entre os níveis de descrição Nobrade e Sepiades (Quadro 1).

Quadro 1. Correspondência entre os níveis descritivos Nobrade e Sepiades.

\begin{tabular}{|l|c|l|}
\hline \multicolumn{1}{|c|}{ Nobrade } & Nível & \multicolumn{1}{c|}{ Sepiades } \\
\hline $\begin{array}{l}\text { Acervo da entidade } \\
\text { custodiadora }\end{array}$ & 0 & Institute \\
\hline Fundo ou Coleção & 1 & Acquisition \\
\hline Seção & 2 & Collection \\
\hline Série & 3 & Grouping \\
\hline Dossiê ou Processo & 4 & Subgrouping* \\
\hline Item Documental & 5 & Single item \\
\hline
\end{tabular}

* Trata-se de um subnível, não é nível principal da Sepiades.

No decorrer do estudo, ao se considerar as particularidades da norma e do modelo analisados, e do contexto desse projeto, foram extraídos apenas os elementos obrigatórios propostos pela norma Nobrade, acrescidos dos elementos descritivos considerados essenciais do modelo Sepiades (Quadro 2). 
Quadro 2. Correspondência entre os elementos descritivos obrigatórios da Nobrade e Sepiades.

\begin{tabular}{|c|c|c|}
\hline Elementos Nobrade & Elementos Sepiades & Comentários \\
\hline $\begin{array}{l}\text { Código de referência } \\
\text { (campo obrigatório) }\end{array}$ & $\begin{array}{l}\text { Main reference code } \\
\text { Acquisition code }\end{array}$ & $\begin{array}{l}\text { Na Sepiades refere-se ao código da instituição } \\
\text { Na Sepiades refere-se ao código da aquisição/produtor do } \\
\text { documento }\end{array}$ \\
\hline \multirow{3}{*}{$\begin{array}{l}\text { Título } \\
\text { (campo obrigatório) }\end{array}$} & Name of institute & Na Sepiades refere-se ao nome da instituição \\
\hline & Title & $\begin{array}{l}\text { Na Sepiades refere-se ao título da fotografia. Pode ser o origi- } \\
\text { nal dado pelo autor, ou outro atribuído pelo catalogador/ } \\
\text { descritor }\end{array}$ \\
\hline & $\begin{array}{l}\text { Location (permanent or } \\
\text { temporary) }\end{array}$ & Na Sepiades refere-se à localização física do documento \\
\hline $\begin{array}{l}\text { Data (campo } \\
\text { obrigatório) }\end{array}$ & Date & $\begin{array}{l}\text { Na Sepiades pode se referir à data da imagem, do } \\
\text { processamento, da descrição, de exposição ou publicação, e } \\
\text { outras... }\end{array}$ \\
\hline $\begin{array}{l}\text { Nível de descrição } \\
\text { (campo obrigatório) }\end{array}$ & & Trata-se da descrição do item documental. \\
\hline \multirow{4}{*}{$\begin{array}{l}\text { Dimensão e suporte } \\
\text { (campo obrigatório) }\end{array}$} & Dimensions & \\
\hline & Photographic type & \multirow{3}{*}{$\begin{array}{l}\text { Os elementos sepiades são individualizados enquanto que a } \\
\text { Nobrade prevê uma descrição mais genérica e textual }\end{array}$} \\
\hline & File format & \\
\hline & Technical identification & \\
\hline $\begin{array}{l}\text { Nome do(s) Produtor(es) } \\
\text { (campo obrigatório) }\end{array}$ & Creator & $\begin{array}{l}\text { Identificação do produtor ou criador. Pode ser a instituição } \\
\text { ou o fotógrafo }\end{array}$ \\
\hline $\begin{array}{l}\text { Condições de acesso } \\
\text { (campo obrigatório) }\end{array}$ & Access restriction & $\begin{array}{l}\text { Na Nobrade esse campo é obrigatório apenas nos níveis } 0 \text { e } \\
1 \text {. }\end{array}$ \\
\hline \multirow[t]{6}{*}{ Âmbito e conteúdo } & Description & Descrição \\
\hline & Contents of acquisition & Conteúdo \\
\hline & Names & Nomes \\
\hline & Geographical location & Na Sepiades refere-se ao local onde a imagem foi obtida \\
\hline & $\begin{array}{l}\text { Origins of collection/ } \\
\text { grouping }\end{array}$ & Origem \\
\hline & $\begin{array}{l}\text { Contents of the } \\
\text { collection/ grouping/ } \\
\text { acquisition }\end{array}$ & Conteúdo \\
\hline Condições de reprodução & Copyright & Direitos autorais não estão previstos na Nobrade \\
\hline $\begin{array}{l}\text { Unidades de descrição } \\
\text { relacionadas }\end{array}$ & Relationships & $\begin{array}{l}\text { O relacionamento deste com outros documentos na mesma } \\
\text { instituição ou em outras, no mesmo acervo ou em outro den- } \\
\text { tro da mesma instituição }\end{array}$ \\
\hline Nota do Arquivista & References & Referencias utilizadas na elaboração da descrição \\
\hline $\begin{array}{l}\text { Pontos de acesso e } \\
\text { indexação de assuntos }\end{array}$ & $\begin{array}{l}\text { Descriptors/subject } \\
\text { headings/classification }\end{array}$ & Descritores propriamente dito \\
\hline
\end{tabular}

Esse estudo de equivalência forneceu subsídios para concluir que ambas as referências utilizadas, baseadas na $\operatorname{ISAD}(G)$, são equivalentes e seus elementos são passíveis de miscigenação. 


\section{RESULTADOS OBTIDOS}

A partir da proposta de elementos descritivos no nível Single item do modelo Sepiades e do conjunto de elementos da Nobrade os elementos descritivos foram sistematizados em quatro conjuntos nomeados como dados administrativos, dados de proveniência, dados técnicos do suporte e dados da imagem (Quadro 3).

Os dados administrativos reúnem elementos que registram informação essencial para identificar a unidade de descrição; sobre como, quando e por quem a descrição foi elaborada; se registra informação sobre outras fontes que têm importante relação com a unidade de descrição e o acompanhamento das disseminações realizadas.

conjunto dos dados de proveniência tem por objetivo contextualizar a origem e produção do documento de acordo com a estrutura de níveis propostos na Nobrade. Considerando que não são obrigatórios todos os níveis, mas são imprescindíveis os níveis instituição, fundo e dossiê, podem juntar-se a esses os níveis série e item. Registra-se a informação sobre a proveniência e a custódia da unidade de descrição.

Os dados técnicos do suporte (Imagem Física na Sepiades) reúnem os elementos descritivos que se referem ao suporte tradicional, aqui chamado fotografia, e aos suportes digitais ou eletrônicos, que na Sepiades é chamado de digital foto file, e que não foram abordados neste momento do estudo. Também é registrada informação sobre o estado de conservação.

Os dados da Imagem (Imagem Visual na Sepiades) englobam os elementos descritivos da Sepiades no nível Single item, e, também, o elemento descritivo Dimensão Expressiva proposto por Manini (2002). Registram-se os termos selecionados para localização e recuperação da unidade de descrição.

Quadro 3. Proposição de metadados para descrição de arquivos fotográficos.

\begin{tabular}{|l|l|}
\hline Dados Administrativos & $\begin{array}{l}\text { código, tipo código, código(s) relacionado(s), denominação, local de guarda, guia de } \\
\text { recolhimento, data recolhimento, Difusão, nome do arquivista e data da descrição. }\end{array}$ \\
\hline Dados de Proveniência & Fundo, grupo, série, dossiê, item, nota explicativa \\
\hline Dados Técnicos do Suporte & $\begin{array}{l}\text { data do processamento, fotógrafo, tradição documental, nitidez, polaridade, dimensão, } \\
\text { cromia, conservação, formato e material. }\end{array}$ \\
\hline Dados da lmagem & $\begin{array}{l}\text { título, tipo do título, nome(s) personagem(ns), local, data, dimensão expressiva, conteú- } \\
\text { do informacional e termos relacionados à imagem }\end{array}$ \\
\hline
\end{tabular}

\section{CONCLUSÃO}

Considerando que as necessidades do usuário desse tipo de acervo são similares em relação ao contexto em que a imagem está inserida e que há várias interpretações possíveis para uma mesma imagem, o desafio da descrição continua presente. Não se esgotam os esforços em direção à padronização visando ao intercâmbio multiinstitucional dessas informações e documentos. Difundir o acervo fotográfico será sempre um desafio para qualquer instituição e disponibilizar a sua documentação fotográfica para um contingente cada vez maior e mais exigente de usuários requer esforços constantes. Pessoal capacitado e treinado e recursos orçamentários são necessários para sustentar e qualificar a oferta desse serviço para os mais diversos perfis de usuários.

Neste sentido, os esforços do Departamento de Arquivo Geral da UFSM são permanentes. Muito já foi realizado para recuperar e proteger a documentação fotográfica dos danos causados pela exposição, acondicionamento e manuseio inadequados. Esse acervo tem sido utilizado por acadêmicos de vários cursos da instituição e de outras universidades para realização de monografias, dissertações e teses. Mas há ainda um vasto território a ser explorado e as possibilidades de disseminação se ampliam com os recursos e as tecnologias disponíveis. A produção de um catálogo impresso e uma versão a ser disponibilizada na web foi o motivador da busca por 
um instrumento descritivo que possibilitasse também a gestão do arquivo fotográfico da UFSM.

A partir desse conjunto de elementos descritivos é possível contextualizar o acervo do qual o documento fotográfico faz parte e disponibilizar informações consistentes e abrangentes ao usuário. Dessa maneira, também é possível realizar uma prática de difusão do arquivo da UFSM.

O que se pretende agora é a aplicação e avaliação desse conjunto de metadados no arquivo permanente da UFSM.

Para concluir se reforça que a produção documental de uma instituição (todas as administrações públicas) deve ser entendida sempre no seu sentido

\section{REFERÊNCIAS}

BRASIL. Conselho Nacional de Arquivos. NOBRADE: Norma Brasileira de Descrição Arquivística. Rio de Janeiro: Arquivo Nacional, 2006.

GENERAL international standard archival description - ISAD(G). 2.ed. OHtawa, 2000. Disponível em: <http://www.ica.org/biblio/ cds/isad_g_2e.pdf > . Acesso em: 10 jan. 2008.

MANINI, M. P. Análise documentária de fotografias: um referencial de leitura de imagens fotográficas para fins documentários. 2002. 231f. Tese (doutorado em Ciência da Informação) - Escola de Comunicações e Artes, Universidade de São Paulo, 2002. integral, porque, a princípio, todo documento, qualquer que seja o suporte em que foi produzido durante o exercício da função pública é arquivístico, e, como tal, se encontra protegido pelas leis de arquivo e patrimônio em âmbito nacional e internacional.

Deve se considerar ainda que na perspectiva da história, os historiadores do futuro terão uma outra noção da importância dos arquivos informáticos que tenham sido perdidos ou destruídos hoje. O descarte indiscriminado da documentação arquivística em meio informático ou o descaso em relação à sua conservação e preservação poderão privar os futuros historiadores da matéria-prima indispensável à reconstituição de uma era que se convencionou denominar como era da informação.
SAFEGUARDING european photographic images for acess SEPIA. 2000. Disponível em: <http://www.knaw.nl/ecpa/sepia/ >. Acesso em: 10 jan. 2008.

UNIVERSIDADE FEDERAL DE SANTA MARIA. Projeto institucional: reestruturação da Coordenadoria de Comunicação Social. Santa Maria, 1998.

UNIVERSIDADE FEDERAL DE SANTA MARIA. Proposta de Regimento interno Arquivo Geral: disposições gerais. Santa Maria, 2001. cap. 5.

UNIVERSIDADE FEDERAL DE SANTA MARIA. Resolução $N^{\circ}$ $0007 / 90$. Altera o item I da Resolução No 0006/90 que trata da transformação da Divisão de Serviços Auxiliares em Divisão de Arquivo Geral. Santa Maria, 1990. 
